\title{
Hosoya Polynomials of Generalized Vertex Identified and Edge Introducing Graphs
}

\author{
Ahmed M. Ali \\ ahmedgraph@uomosul.edu.iq \\ Noor M. Dahash \\ College of Computer Science and Mathematics \\ University of Mosul, Mosul, Iraq
}

Received on: 13/05/2012

Accepted on: 18/09/2012

\begin{abstract}
The vertex identified and edge introducing graphs of two disjoint connected graphs are extended to $\mathrm{n}, n \geq 3$, disjoint connected graphs. Hosoya polynomials and Wiener indices of such composite graphs are obtained by applying Gutman's Theorem and using mathematical induction on $\mathrm{n}$.
\end{abstract}

Keywords: distance, Hosoya Polynomial, Wiener index.

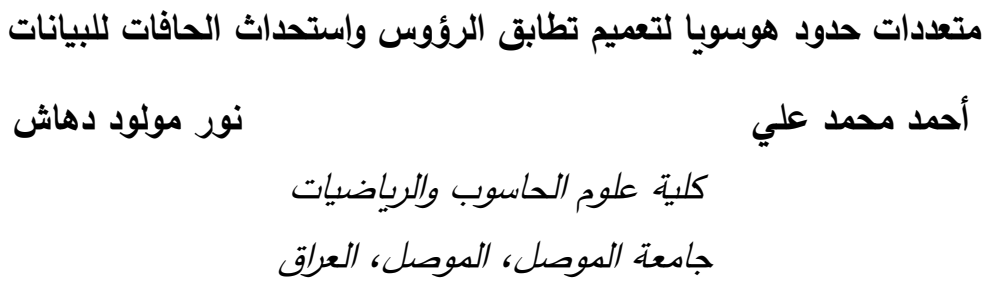

تاريخ قبول البحث: 2012/09/18

تاريخ استلام البحث: 2012/05/13

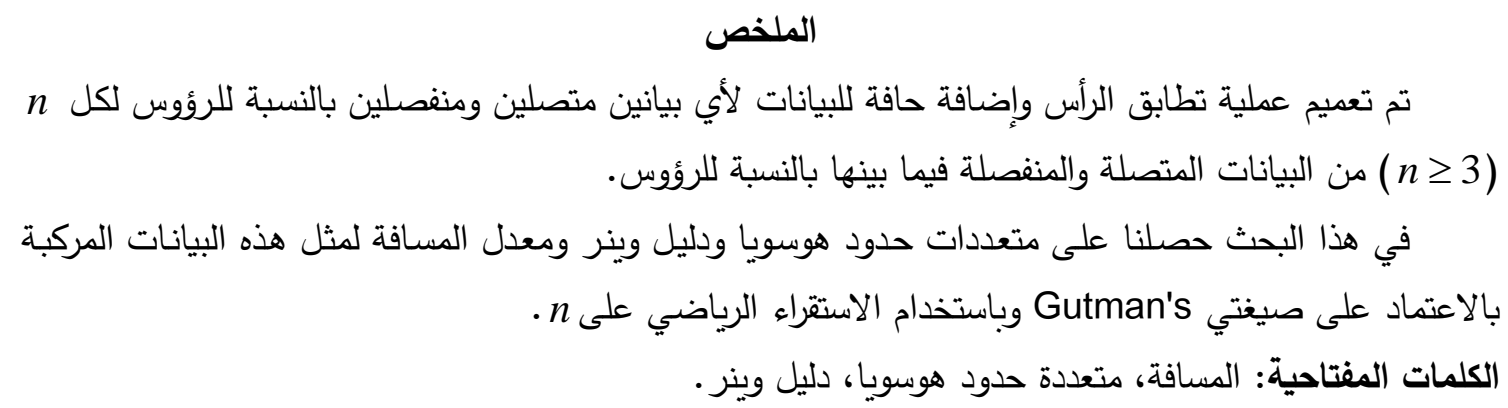

\section{Introduction}

Our terminology and notations will be as given in the references [2,3,5].

Let $G$ be a connected graph of order $p$ and size $q$. The distance between the two vertices $u$ and $v$ in $G$ is the minimum of the lengths of $u-v$ paths in $G$;it is denoted by $d_{G}(u, v)$ or simply $d(u, v)$.

The eccentricity $e(v)$ of a vertex $v$ in $G$ is defined by $e(v)=\max \{d(u, v): u \in V(G)\}$,

Where,$V(G)$ is the vertex set of $G$. The diameter of $G$ denoted by $\operatorname{diam} G$, or $\delta$, is defined by:

$\operatorname{diam} G=\max \{e(v): v \in V(G)\}=\max \{d(u, v): u, v \in V(G)\}$. 
The concept of distance based polynomial was introduced in 1988 by $\mathrm{H}$. Hosoya[7]. Hosoya's paper, which seems to be the only published work concerning this issue, reported a limited number of results on Hosoya polynomials.

Let $d(G, k)$ be the number of pairs of vertices in $G$ that are at distance $k$ apart , $0 \leq k \leq \delta$, then the Hosoya polynomial of a connected graph $G$ is defined as follows : $H(G ; x)=\sum_{k=0}^{\delta} d(G, k) x^{k}$.

The number of all pairs of vertices $u$ and $v$ in $G$ is $p(p+1) / 2$, where $p$ is the order of $G$.

Let $d(v, G, k)$ be the number of vertices in $G$, that are at distance k from vertex $v$, then the Hosoya polynomial of a vertex $v$ is defined by [4]

$H(v, G ; x)=\sum_{k \geq 0} d(v, G, k) x^{k}$.

Observe that

$$
\sum_{v \in V(G)} d(v, G, k)=\left\{\begin{array}{l}
p, \text { if } k=0 \\
2 d(G, k), \text { if } k>0
\end{array}\right.
$$

From this, we have a relation between the Hosoya polynomial of $G$ and Hosoya polynomial of a vertex $\mathrm{v}$ in $G$, namely,

$\sum_{v \in V(G)} H(v, G ; x)=2 H(G ; x)-p$.

Several authers had obtained Hosoya polynomials for special graphs and compound graphs obtained by certain graph binary operations [7,8,10,11]. In 1993, Gutman [6] defined vertex identified and edge introducing graphs, constructed from two vertex disjoint connected graphs as given next:

\section{Definition 1.1:[6]}

Let $G_{1}$ and $G_{2}$ be any two connected graphs with disjoint vertex sets. Let $\mathrm{u}$ and $\mathrm{v}$ be any two vertices of $G_{1}$ and $G_{2}$ respectively, then the vertex identified graph $G_{1} \bullet G_{2}$ is obtained from $G_{1}$ and $G_{2}$ by identifying the vertices $u$ and $v$, the edge introducing graph $G_{1}: G_{2}$ is the graph obtained from $G_{1}$ and $G_{2}$ by introducing a new edge joining the vertices $u$ and $v$. Gutman obtained $H\left(G_{1} \bullet G_{2} ; x\right)$ and $H\left(G_{1}: G_{2} ; x\right)$ as follows:

\section{Gutman's Theorem 1.2:[6]}

1. $H\left(G_{1} \bullet G_{2} ; x\right)=H\left(G_{1} ; x\right)+H\left(G_{2} ; x\right)+H\left(u, G_{1} ; x\right) H\left(v, G_{2} ; x\right)$ $-H\left(u, G_{1} ; x\right)-H\left(v, G_{2} ; x\right)$.

2. $H\left(G_{1}: G_{2} ; x\right)=H\left(G_{1} ; x\right)+H\left(G_{2} ; x\right)+x H\left(u, G_{1} ; x\right) H\left(v, G_{2} ; x\right)$.

In this paper, we continue the research along the same lines to find Hosoya polynomials and Wiener indices of graphs constructed from several vertex disjoint graphs by using the idea of vertex identification and edge introducing.

\section{Generalized Vertex Identified Graphs:}

Let $G_{1}, G_{2}, \ldots, G_{n}, n \geq 2$, be disjoint connected graphs and let $u_{i}, v_{i} \in V\left(G_{i}\right)$ $\forall i=1,2, \ldots, n$.Then,$G_{1} \bullet G_{2} \bullet \ldots \bullet G_{n}$ is the graph obtained from $G_{1}, G_{2}, \ldots, G_{n}$ by 
identifying the vertices $u_{i}$ with $v_{i+1}$, for all $i=1,2, \ldots, n-1$. (See Fig.2.1). To simplify the notation, we denote $G_{1} \bullet G_{2} \bullet \ldots \bullet G_{n}$ by $\prod_{i=1}^{n}\left(G_{i}, \bullet\right)$ or $J_{n}$.

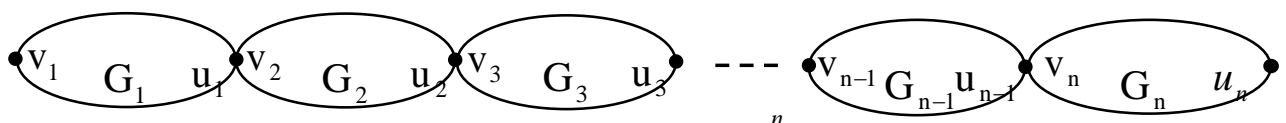

\section{Fig. 2.1. The graph $\prod_{i=1}^{n}\left(G_{i}, \bullet\right)$}

It is clear that $p\left(J_{n}\right)=\sum_{i=1}^{n} p\left(G_{i}\right)-n+1 \quad, \quad q\left(J_{n}\right)=\sum_{i=1}^{n} q\left(G_{i}\right), \quad$ and $\operatorname{diam}\left(J_{n}\right)=\max _{i<j}\left\{e_{G_{i}}\left(u_{i}\right)+e_{G_{j}}\left(v_{j}\right)+\sum_{r=i+1}^{j-1} d\left(u_{r}, v_{r}\right)\right\}$.

In the next theorem, we give the Hosoya polynomial of $J_{n}$ for all $n \geq 2$.

Theorem 2.1: For any positive integer $n, n \geq 2$, we have.

$H\left(J_{n} ; x\right)=\sum_{i=1}^{n} H\left(G_{i} ; x\right)-\sum_{i=2}^{n} H\left(v_{i}, G_{i} ; x\right)+\sum_{i=1}^{n-1} H\left(u_{i}, J_{i} ; x\right)\left[H\left(v_{i+1}, G_{i+1} ; x\right)-1\right]$.

Proof: The formula (2.1) will be proved by mathematical induction on $\mathrm{n}, n \geq 2$.

For $\mathrm{n}=2$, we have by Gutman's Theorem ,

$$
\begin{aligned}
H\left(G_{1} \bullet G_{2} ; x\right) & =H\left(G_{1} ; x\right)+H\left(G_{2} ; x\right)+H\left(u_{1}, G_{1} ; x\right) H\left(v_{2}, G_{2} ; x\right) \\
& -H\left(u_{1}, G_{1} ; x\right)-H\left(v_{2}, G_{2} ; x\right)
\end{aligned}
$$

Thus, (2.1) is true for $n=2$.

We assume that (2.1) is true for $n=r \geq 2$ and prove it for $n=r+1$.

Then, for the graph using Gutman's Theorem, we get

$$
\begin{aligned}
H\left(J_{r+1} ; x\right) & =H\left(J_{r} \bullet G_{r+1} ; x\right)=H\left(J_{r} ; x\right)+H\left(G_{r+1} ; x\right)+H\left(u_{r}, J_{r} ; x\right) H\left(v_{r+1}, G_{r+1} ; x\right) \\
& -H\left(u_{r}, J_{r} ; x\right)-H\left(v_{r+1}, G_{r+1} ; x\right) \\
& =\sum_{i=1}^{r+1} H\left(G_{i} ; x\right)-\sum_{i=2}^{r+1} H\left(v_{i}, G_{i} ; x\right)+\sum_{i=1}^{r} H\left(u_{i}, J_{i} ; x\right)\left[H\left(v_{i+1}, G_{i+1} ; x\right)-1\right]
\end{aligned}
$$

Hence, (2.1) is true for all $\mathrm{n}, n \geq 2$.

Lemma 2.2: For all $u_{i}, v_{i} \in V\left(G_{i}\right), i=1,2, \ldots, m, m \geq 2$, we have.

$$
H\left(u_{m}, J_{m} ; x\right)=H\left(u_{m}, G_{m} ; x\right)+\sum_{j=1}^{m-1}\left(H\left(u_{j}, G_{j} ; x\right)-1\right) x^{\sum_{h=j+1}^{m} d\left(u_{h}, v_{h}\right)} \text {. }
$$

Proof: We use mathematical induction on $\mathrm{m}, \mathrm{m} \geq 2$.

From Fig.2.1, we notice that, when $\mathrm{m}=2$

$$
H\left(u_{2}, G_{1} \bullet G_{2} ; x\right)=H\left(u_{2}, G_{2} ; x\right)+\left[H\left(u_{1}, G_{1} ; x\right)-1\right] x^{d\left(u_{2}, v_{2}\right)}
$$

Thus , (2.2) is true for $\mathrm{m}=2$.

Now, we assume that (2.2) is true for all $r \geq 2$.

For $r+1$, we have

$$
\begin{aligned}
H\left(u_{r+1}, J_{r+1} ; x\right) & =H\left(u_{r+1}, G_{r+1} ; x\right)+\left[H\left(u_{r}, J_{r} ; x\right)-1\right] x^{d\left(u_{r+1}, v_{r+1}\right)} . \\
= & H\left(u_{r+1}, G_{r+1} ; x\right)+\left[H\left(u_{r}, G_{r} ; x\right)+\sum_{j=1}^{r-1}\left(H\left(u_{j}, G_{j} ; x\right)-1\right) x^{\sum_{h=j+1}^{r} d\left(u_{h}, v_{h}\right)}-1\right] x^{d\left(u_{r+1}, v_{r+1}\right)}
\end{aligned}
$$




$$
=H\left(u_{r+1}, G_{r+1} ; x\right)+\sum_{j=1}^{r}\left(H\left(u_{j}, G_{j} ; x\right)-1\right) x^{\sum_{h=j+1}^{r+1} d\left(u_{h}, v_{h}\right)}
$$

Hence, (2.2) is true for all $\mathrm{m}, \mathrm{m} \geq 2$.

From Theorem 2.1 and Lemma 2.2 , we obtain the following theorem.

Theorem 2.3: For any positive integer $\mathrm{n}, n \geq 3$, we have

$$
\begin{aligned}
H\left(J_{n} ; x\right) & =\sum_{i=1}^{n} H\left(G_{i} ; x\right)+\sum_{i=1}^{n-1}\left[\left(H\left(u_{i}, G_{i} ; x\right)-1\right)\left(H\left(v_{i+1}, G_{i+1} ; x\right)-1\right)-1\right] \\
& +\sum_{i=2}^{n-1} \sum_{j=1}^{i-1}\left[H\left(u_{j}, G_{j} ; x\right)-1\right]\left[H\left(v_{i+1}, G_{i+1} ; x\right)-1\right] x^{\sum_{h=j+1}^{i} d\left(u_{h}, v_{h}\right)} .
\end{aligned}
$$

Proof: From Theorem 2.1, we have

$$
\begin{aligned}
H\left(J_{n} ; x\right) & =\sum_{i=1}^{n} H\left(G_{i} ; x\right)-\sum_{i=2}^{n} H\left(v_{i}, G_{i} ; x\right)+H\left(u_{1}, G_{1} ; x\right)\left[H\left(v_{2}, G_{2} ; x\right)-1\right] \\
& +\sum_{\mathrm{i}=2}^{\mathrm{n}-1} \mathrm{H}\left(\mathrm{u}_{\mathrm{i}}, \mathrm{J}_{\mathrm{i}} ; \mathrm{x}\right)\left[\mathrm{H}\left(\mathrm{v}_{\mathrm{i}+1}, \mathrm{G}_{\mathrm{i}+1} ; \mathrm{x}\right)-1\right]
\end{aligned}
$$

Then From (2.2), we get

$$
\begin{aligned}
H\left(J_{n} ; x\right) & =\sum_{i=1}^{n} H\left(G_{i} ; x\right)-\sum_{i=2}^{n} H\left(v_{i}, G_{i} ; x\right)+H\left(u_{1}, G_{1} ; x\right)\left[H\left(v_{2}, G_{2} ; x\right)-1\right] \\
& +\sum_{i=2}^{n-1} H\left(u_{i}, G_{i} ; x\right)\left[H\left(v_{i+1}, G_{i+1} ; x\right)-1\right] \\
& +\sum_{i=2}^{n-1} \sum_{j=1}^{i-1}\left[H\left(u_{j}, G_{j} ; x\right)-1\right]\left[H\left(v_{i+1}, G_{i+1} ; x\right)-1\right] x^{\sum_{h=j+1}^{i} d\left(u_{h}, v_{h}\right)} .
\end{aligned}
$$

Simplifying the last expression, we obtain (2.3).

If each of $G_{1}, G_{2}, \ldots, G_{n}$ is isomorphic to $\mathrm{G}$, then $G \bullet G \bullet \ldots \bullet G$ is denoted by $J_{n}(G)$. It is obvious that $p\left(J_{n}(G)\right)=n p(G)-n+1, q\left(J_{n}(G)\right)=n q(G)$, and if $u_{i}=u$ ,$v_{i}=v$, for all $i=1,2, \ldots, n$, then $\operatorname{diam}\left(J_{n}(G)\right)=e_{G}(u)+e_{G}(v)+(n-2) d_{G}(u, v)$.

If $d_{G}(u, v)=\operatorname{diam} G$, then $\operatorname{diam}\left(J_{n}(G)\right)=n \operatorname{diam} G$.

Corollary 2.4: If $\mathrm{G}$ is a connected graph and $d_{G}(u, v)=\operatorname{diam} G=\delta$, then for any positive integer $\mathrm{n}, n \geq 3$, we have

$$
\begin{aligned}
H\left(J_{n}(G) ; x\right)= & n H(G ; x)+(n-1)[(H(u, G ; x)-1)(H(v, G ; x)-1)-1] \\
& +(H(u, G ; x)-1)(H(v, G ; x)-1) \sum_{i=2}^{n-1} \sum_{j=1}^{i-1} x^{(i-j) \delta} .
\end{aligned}
$$

Corollary 2.5: If $\mathrm{G}$ is a connected graph and $d_{G}(u, v)=\operatorname{diam} G=\delta$ with $H(u, G ; x)=H(v, G ; x)$, then for any positive integer $\mathrm{n}, n \geq 3$, we have

$$
\begin{aligned}
H\left(J_{n}(G) ; x\right)= & \left.n H(G ; x)+(n-1)\left[(H(u, G ; x)-1)^{2}-1\right)\right] \\
& +(H(u, G ; x)-1)^{2} \sum_{i=2}^{n-1} \sum_{j=1}^{i-1} x^{(i-j) \delta} .
\end{aligned}
$$




\section{Generalized Edge Introducing Graphs}

Let $G_{1}, G_{2}, \ldots, G_{n}$ be disjoint connected graphs and let $u_{i}, v_{i} \in V\left(G_{i}\right)$, $\forall i=1,2, \ldots, n$, then the edge introduce graph $G_{1}: G_{2}: \ldots: G_{n}$ is obtained from $G_{1}, G_{2}, \ldots, G_{n}$ by adding new edge $u_{i} v_{i+1}$, for all $i=1,2, \ldots, n-1$.( See Fig. 3.1).

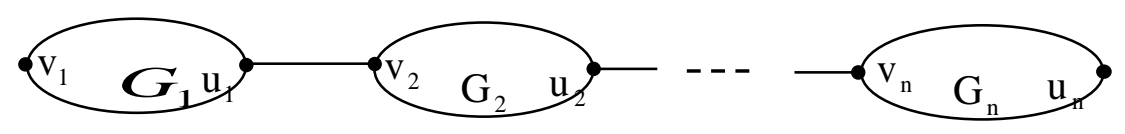

Fig. 3.1. The graph $\prod_{i=1}^{n}\left(G_{i},:\right)$

To simplify our notation, we denote $G_{1}: G_{2}: \ldots: G_{n}$ by $\prod_{i=1}^{n}\left(G_{i},:\right)$ or $\mathrm{I}_{n}$.

It is clear that $p\left(I_{n}\right)=\sum_{i=1}^{n} p\left(G_{i}\right), \quad q\left(I_{n}\right)=\sum_{i=j}^{n} q\left(G_{i}\right)+n-1, \quad$ and $\operatorname{diam}\left(I_{n}\right)=\max _{i<j}\left\{e_{G_{i}}\left(u_{i}\right)+e_{G_{j}}\left(v_{j}\right)+\sum_{r=i+1}^{j-1} d_{G_{r}}\left(u_{r}, v_{r}\right)+j-i\right\}$.

Theorem 3.1: For any positive integer $\mathrm{n}, n \geq 2$, the Hosoya polynomial of $\mathrm{I}_{n}$ is given by.

$$
H\left(I_{n} ; x\right)=\sum_{i=1}^{n} H\left(G_{i} ; x\right)+x \sum_{i=1}^{n-1} H\left(u_{i}, I_{i} ; x\right) H\left(v_{i+1}, G_{i+1} ; x\right) \text {. }
$$

Proof: We use mathematical induction on $\mathrm{n}, n \geq 2$.

For $\mathrm{n}=2$, we have, by Gutman's Theorem, that

$$
H\left(G_{1}: G_{2} ; x\right)=H\left(G_{1} ; x\right)+\left(G_{2} ; x\right)+x H\left(u_{1}, G_{1} ; x\right) H\left(v_{2}, G_{2} ; x\right)
$$

Thus, (3.1) is true for $\mathrm{n}=2$.

We assume that (3.1) is true for $n=r \geq 2$, and prove that it is true for $\mathrm{n}=\mathrm{r}+1$.

$$
\begin{aligned}
H\left(I_{r+1} ; x\right) & =H\left(I_{r}: G_{r+1} ; x\right)=H\left(I_{r} ; x\right)+H\left(G_{r+1} ; x\right)+x H\left(u_{r}, I_{r} ; x\right) H\left(v_{r+1}, G_{r+1} ; x\right) \\
& =\sum_{i=1}^{r} H\left(G_{i} ; x\right)+x \sum_{i=1}^{r-1} H\left(u_{i}, I_{i} ; x\right) H\left(v_{i+1}, G_{i+1} ; x\right)+H\left(G_{r+1} ; x\right) \\
& +x H\left(u_{r}, I_{r} ; x\right) H\left(v_{r+1}, G_{r+1} ; x\right) \\
& =\sum_{i=1}^{r+1} H\left(G_{i} ; x\right)+x \sum_{i=1}^{r} H\left(u_{i}, I_{i} ; x\right) H\left(v_{i+1}, G_{i+1} ; x\right) .
\end{aligned}
$$

Hence, (3.1) is true for all $n \geq 2$.

Lemma 3.2: For all $m \geq 2$, we have

$$
H\left(u_{m}, I_{m} ; x\right)=H\left(u_{m}, G_{m} ; x\right)+\sum_{j=1}^{m-1} H\left(u_{j}, G_{j} ; x\right) x^{\sum_{h=j+1}^{m} d\left(u_{h}, v_{h}\right)+(m-j)} .
$$

Proof: When $\mathrm{m}=2$, we have.

$$
H\left(u_{2}, I_{2} ; x\right)=H\left(u_{2}, G_{2} ; x\right)+H\left(u_{1}, G_{1} ; x\right) x^{d\left(u_{2}, v_{2}\right)+1}
$$

Now, we assume that (3.2) is true for $r \geq 2$, and show that it is true for $r+1$, $H\left(u_{r+1}, I_{r+1} ; x\right)=H\left(u_{r+1}, G_{r+1} ; x\right)+H\left(u_{r}, I_{r} ; x\right) x^{d\left(u_{r+1}, v_{r+1}\right)+1}$ 


$$
\begin{aligned}
& =H\left(u_{r+1}, G_{r+1} ; x\right)+\left[H\left(u_{r}, G_{r} ; x\right)+\sum_{j=1}^{r-1} H\left(u_{j}, G_{j} ; x\right) x^{\sum_{h=j+1}^{r} d\left(u_{h}, v_{h}\right)+(r-j)}\right] x^{d\left(u_{r+1}, v_{r+1}\right)+1} \\
& =H\left(u_{r+1}, G_{r+1} ; x\right)+\sum_{j=1}^{r+1} H\left(u_{j}, G_{j} ; x\right) x^{h=j+1} d\left(u_{h}, v_{h}\right)+(r+1-j)
\end{aligned}
$$

Hence, (3.2) is true for all $m \geq 2$.

Now, using Theorem 3.1 and Lemma 3.2, we prove the following theorem .

Theorem 3.3: For any positive integer $\mathrm{n}, n \geq 3$, we have.

$$
\begin{aligned}
H\left(I_{n} ; x\right) & =\sum_{i=1}^{n} H\left(G_{i} ; x\right)+x \sum_{i=1}^{n-1} H\left(u_{i}, G_{i} ; x\right) H\left(v_{i+1}, G_{i+1} ; x\right) \\
& +\sum_{i=2}^{n-1} \sum_{j=1}^{i-1} H\left(u_{j}, G_{j} ; x\right) H\left(v_{i+1}, G_{i+1} ; x\right) x^{\sum_{h=j+1}^{i} d\left(u_{h}, v_{h}\right)+(i+1-j)} .
\end{aligned}
$$

Proof: From Theorem 3.1, we have

$$
\begin{aligned}
H\left(I_{n} ; x\right) & =\sum_{i=1}^{n} H\left(G_{i} ; x\right)+x H\left(u_{1}, G_{1} ; x\right) H\left(v_{2}, G_{2} ; x\right) \\
& +x \sum_{i=2}^{n-1} H\left(u_{i}, I_{i} ; x\right) H\left(v_{i+1}, G_{i+1} ; x\right),
\end{aligned}
$$

Then, from Lemma 3.2,we obtain

$$
\begin{aligned}
& H\left(I_{n} ; x\right)=\sum_{i=1}^{n} H\left(G_{i} ; x\right)+x H\left(u_{1}, G_{1} ; x\right) H\left(v_{2}, G_{2} ; x\right) \\
& +x \sum_{i=2}^{n-1}\left[H\left(u_{i}, G_{i} ; x\right)+\sum_{j=1}^{i-1} H\left(u_{j}, G_{j} ; x\right) x^{\sum_{h=j+1}^{i} d\left(u_{h}, v_{h}\right)+(i-j)}\right] H\left(v_{i+1}, G_{i+1} ; x\right) \\
& =\sum_{i=1}^{n} H\left(G_{i} ; x\right)+x \sum_{i=1}^{n-1} H\left(u_{i}, G_{i} ; x\right) H\left(v_{i+1}, G_{i+1} ; x\right) \\
& +\sum_{i=2}^{n-1} \sum_{j=1}^{i-1} H\left(u_{j}, G_{j} ; x\right) H\left(v_{i+1}, G_{i+1} ; x\right) x^{\sum_{h=j+1}^{i} d\left(u_{h}, v_{h}\right)+(i+1-j)} .
\end{aligned}
$$

This completes the proof .

Now, if each of $G_{1}, G_{2}, \ldots, G_{n}$ is isomorphic to $\mathrm{G}$, then $G: G: \ldots: G$ is denoted by $I_{n}(G)$. It is clear that $p\left(I_{n}(G)\right)=n p(G), q\left(I_{n}(G)\right)=n q(G)+n-1$, and if $u_{i}=u$, $v_{i}=v$, for all $i=1,2, \ldots, n$, then $\operatorname{diam}\left(\mathrm{I}_{\mathrm{n}}(\mathrm{G})\right)=\mathrm{e}_{\mathrm{G}}(\mathrm{u})+\mathrm{e}_{\mathrm{G}}(\mathrm{v})+(\mathrm{n}-2) \mathrm{d}_{\mathrm{G}}(\mathrm{u}, \mathrm{v})+\mathrm{n}-1$

If $d_{G}(u, v)=\operatorname{diam} G$, then $\operatorname{diam}\left(I_{n}(G)\right)=n \operatorname{diam} G+n-1$.

Corollary 3.4: If $\mathrm{G}$ is a connected graph and $\mathrm{d}_{\mathrm{G}}(\mathrm{u}, \mathrm{v})=\operatorname{diam} \mathrm{G}=\delta$, then for any positive integer $\mathrm{n}, \boldsymbol{n} \geq \mathbf{3}$, we obtain.

$$
\begin{aligned}
H\left(I_{n}(G) ; x\right) & =n H(G ; x)+(n-1) H(u, G ; x) H(v, G ; x) x \\
& +H(u, G ; x) H(v, G ; x) \sum_{i=2}^{n-1} \sum_{j=1}^{i-1} x^{(i-j)(\delta+1)+1} .
\end{aligned}
$$


Corollary 3.5: If $\mathrm{G}$ is a connected graph and $d(u, v)=\operatorname{diam} G=\delta$ with $H(u, G ; x)=H(v, G ; x)$, then for any positive integer $\mathrm{n}, n \geq 3$, we have.

$H\left(I_{n}(G) ; x\right)=n H(G ; x)+(n-1)(H(u, G ; x))^{2} x+(H(u, G ; x))^{2} \sum_{i=2}^{n-1} \sum_{j=1}^{i-1} x^{(i-j)(\delta+1)+1}$

\section{Some Applications of Hosoya Polynomials:}

\subsection{Hosoya Polynomials of Special Vertex Identified Graphs in Chemistry.}

\section{Examples 4.1.1:}

1. If $C_{t}$ is an even cycle graph of order $t, t=2 m, m \geq 2$, then, $\operatorname{diam~} C_{2 m}=m$, $H\left(u, C_{2 m} ; x\right)=1+2 \sum_{r=1}^{m-1} x^{r}+x^{m}$, for all $\mathrm{u} \in \mathrm{V}\left(\mathrm{C}_{2 \mathrm{~m}}\right)$, and $\mathrm{H}\left(\mathrm{C}_{2 \mathrm{~m}} ; \mathrm{x}\right)=2 \mathrm{~m} \sum_{\mathrm{r}=0}^{\mathrm{m}-1} \mathrm{x}^{\mathrm{r}}+\mathrm{mx}^{\mathrm{m}}$.

Then, from Corollary 2.5, we have.

$$
\begin{aligned}
H\left(J_{n}\left(C_{2 m}\right) ; x\right) & =2 m n \sum_{r=0}^{m-1} x^{r}+m n x^{m}+(n-1)\left[\left(2 \sum_{r=1}^{m-1} x^{r}+x^{m}\right)^{2}-1\right]+\left(2 \sum_{r=1}^{m-1} x^{r}+x^{m}\right)^{2} \sum_{i=2}^{n-1} \sum_{j=1}^{i-1} x^{m(i-j)} \\
& =n(2 m-1)+1+2 m n \sum_{r=1}^{m-1} x^{r}+m n x^{m}+(n-1) F(x)+F(x) \sum_{i=2}^{n-1} \sum_{j=1}^{i-1} x^{m(i-j)},
\end{aligned}
$$

Where,

$$
F(x)=4 \sum_{r=1}^{m-1} r x^{r+1}+4 \sum_{r=1}^{m-1}(m-r) x^{m+r}+x^{2 m} .
$$

If we take $\mathrm{t}=4$ and $\mathrm{t}=6$,(see Fig.4.1.2), we get
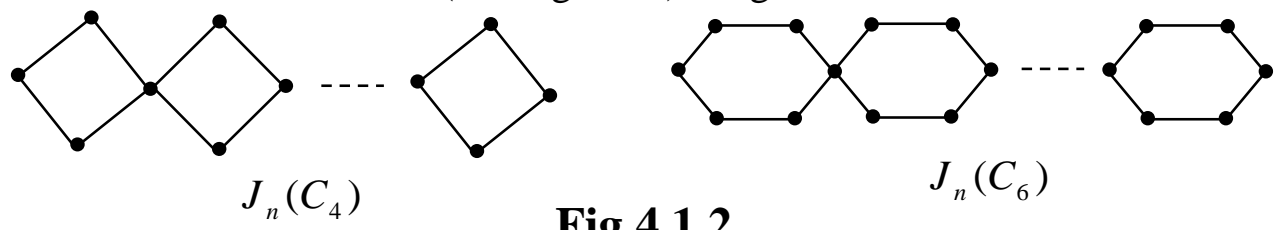

Fig.4.1.2.

$$
\begin{aligned}
H\left(J_{n}\left(C_{4}\right) ; x\right) & =3 n+1+4 n x+2(3 n-2) x^{2}+4(n-1) x^{3}+(n-1) x^{4} \\
& +\left(2 x+x^{2}\right)^{2} \sum_{i=2}^{n-1} \sum_{j=1}^{i-1} x^{2(i-j)}
\end{aligned}
$$

and

$$
\begin{aligned}
H\left(J_{n}\left(C_{6}\right) ; x\right) & =5 n+1+6 n x+2(5 n-2) x^{2}+(11 n-8) x^{3}+(n-1)\left(8 x^{4}+4 x^{5}+x^{6}\right) \\
& +\left(2 x+2 x^{2}+x^{3}\right)^{2} \sum_{i=2}^{n-1} \sum_{j=1}^{i-1} x^{3(i-j)} .
\end{aligned}
$$

Such graphs $J_{n}\left(C_{4}\right)$ and $J_{n}\left(C_{6}\right)$ are used in chemistry.

2. Let $\mathrm{S}_{\mathrm{t}}$ be the star of order $\mathrm{t}, \mathrm{t} \geq 4$, with the vertex set $\mathrm{V}\left(\mathrm{S}_{\mathrm{t}}\right)=\left\{\mathrm{c}, \mathrm{v}_{1}, \mathrm{v}_{2}, \ldots, \mathrm{v}_{\mathrm{t}-1}\right\}$, where $\operatorname{deg} \mathrm{c}=\mathrm{t}-1$ and $\operatorname{deg} \mathrm{v}=1, \forall \mathrm{v} \in \mathrm{V}\left(\mathrm{S}_{\mathrm{t}}\right)-\{\mathrm{c}\}$.

Since, $\quad \operatorname{diam} \mathrm{S}_{\mathrm{t}}=2, \quad \mathrm{H}\left(\mathrm{v}, \mathrm{S}_{\mathrm{t}} ; \mathrm{x}\right)=1+\mathrm{x}+(\mathrm{t}-2) \mathrm{x}^{2}, \quad$ for all $\mathrm{v} \in \mathrm{V}\left(\mathrm{S}_{\mathrm{t}}\right)-\{\mathrm{c}\}$, and $\mathrm{H}\left(\mathrm{S}_{\mathrm{t}} ; \mathrm{x}\right)=\mathrm{t}+(\mathrm{t}-1) \mathrm{x}+\frac{1}{2}(\mathrm{t}-1)(\mathrm{t}-2) \mathrm{x}^{2}$,

then assuming that the vertex of identification of each $S_{t}$ is an end vertex and by using Corollary 2.5, we get 


$$
\begin{aligned}
H\left(J_{n}\left(S_{t}\right) ; x\right) & =n\left[t+(t-1) x+\frac{1}{2}(t-1)(t-2) x^{2}\right] \\
& +(n-1)\left[\left(x+(t-2) x^{2}\right)^{2}-1\right]+\left(x+(t-2) x^{2}\right)^{2} \sum_{i=2}^{n-1} \sum_{j=1}^{i-1} x^{2(i-j)} \\
& =n(t-1)+1+n(t-1) x+\left[\frac{n(t-1)(t-2)}{2}+n-1\right] x^{2} \\
& +2(n-1)(t-2) x^{3}+(n-1)(t-2)^{2} x^{4}+\left(x+(t-2) x^{2}\right)^{2} \sum_{i=2}^{n-1} \sum_{j=1}^{i-1} x^{2(i-j)} .
\end{aligned}
$$

3. Let $\mathrm{W}_{\mathrm{t}}$ be the wheel graph of order $t$, $t \geq 4$, with vertex set $\mathrm{V}\left(\mathrm{W}_{\mathrm{t}}\right)=\left\{\mathrm{c}, \mathrm{v}_{1}, \mathrm{v}_{2}, \ldots, \mathrm{v}_{\mathrm{t}-1}\right\}$, where $\operatorname{deg} \mathrm{c}=\mathrm{t}-1$, and $\operatorname{deg} \mathrm{v}=3, \forall \mathrm{v} \in \mathrm{V}\left(\mathrm{W}_{\mathrm{t}}\right)-\{\mathrm{c}\}$.

Since, $\operatorname{diam} \mathrm{W}_{\mathrm{t}}=2, \mathrm{H}\left(\mathrm{v}, \mathrm{W}_{\mathrm{t}} ; \mathrm{x}\right)=1+3 \mathrm{x}+(\mathrm{t}-4) \mathrm{x}^{2}$, for all $\mathrm{v} \in \mathrm{V}\left(\mathrm{W}_{\mathrm{t}}\right)-\{\mathrm{c}\}$ and $\mathrm{H}\left(\mathrm{W}_{\mathrm{t}} ; \mathrm{x}\right)=\mathrm{t}+2(\mathrm{t}-1) \mathrm{x}+\frac{(\mathrm{t}-1)(\mathrm{t}-4)}{2} \mathrm{x}^{2}$,

Then, assuming that the vertex of identification of each $\mathrm{W}_{t}$ is vertex of degree 3 and by using Corollary 2.5 , we get.

$$
\begin{aligned}
\mathrm{H}\left(\mathrm{J}_{\mathrm{n}}\left(\mathrm{W}_{\mathrm{t}} ; \mathrm{x}\right)\right. & =\mathrm{n}\left[\mathrm{t}+2(\mathrm{t}-1) \mathrm{x}+\frac{(\mathrm{t}-1)(\mathrm{t}-4)}{2} \mathrm{x}^{2}\right]+(\mathrm{n}-1)\left[\left(3 \mathrm{x}+(\mathrm{t}-4) \mathrm{x}^{2}\right)^{2}-1\right] \\
& +\left(3 \mathrm{x}+(\mathrm{t}-4) \mathrm{x}^{2}\right)^{2} \sum_{\mathrm{i}=2}^{\mathrm{n}-1} \sum_{\mathrm{j}=1}^{\mathrm{i}-1} \mathrm{x}^{2(\mathrm{i}-\mathrm{j})} \\
& =\mathrm{n}(\mathrm{t}-1)+1+2 \mathrm{n}(\mathrm{t}-1) \mathrm{x}+\left[\frac{\mathrm{n}(\mathrm{t}-1)(\mathrm{t}-4)}{2}+9(\mathrm{n}-1)\right] \mathrm{x}^{2} \\
& +6(\mathrm{n}-1)(\mathrm{t}-4) \mathrm{x}^{3}+(\mathrm{n}-1)(\mathrm{t}-4)^{2} \mathrm{x}^{4}+\left(3 \mathrm{x}+(\mathrm{t}-4) \mathrm{x}^{2}\right)^{2} \sum_{\mathrm{i}=2}^{\mathrm{n}-1} \sum_{\mathrm{j}=1}^{\mathrm{i}-1} \mathrm{x}^{2(\mathrm{i}-\mathrm{j})}
\end{aligned}
$$

\subsection{Hosoya Polynomials of Special Edge Introducing Graphs}

To illustrate the usefulness of Corollary 3.5,we take G to be a special graph ,such as complete graph, even cycle, star or wheel.

\section{Examples 4.2.1:}

1. If $C_{2 m}, \mathrm{~m} \geq 2$ is an even cycle then, by Corollary 3.5 ,we get

$$
\begin{aligned}
H\left(I_{n}\left(C_{2 m}\right) ; x\right) & =2 m n \sum_{r=0}^{m-1} x^{r}+m n x^{m}+(n-1)\left(1+2 \sum_{r=1}^{m-1} x^{r}+x^{m}\right)^{2} x \\
& +\left(1+2 \sum_{r=1}^{m-1} x^{r}+x^{m}\right)^{2} \sum_{i=2}^{n-1} \sum_{j=1}^{i-1} x^{(i-j)(m+1)+1} \\
& =2 m n \sum_{r=0}^{m-1} x^{r}+m n x^{m}+(n-1) F(x) x+F(x) \sum_{i=2}^{i-1} \sum_{j=1}^{i-1} x^{(i-j)(m+1)+1},
\end{aligned}
$$

Where ,

$$
F(x)=4 \sum_{r=1}^{m-1}(r x+1) x^{r}+4 \sum_{r=1}^{m-1}(m-r) x^{m+r}+\left(1+x^{m}\right)^{2}
$$

For special cases, we take $\mathrm{t}=4$ and $\mathrm{t}=6$ then ,

$$
H\left(I_{n}\left(C_{4}\right) ; x\right)=4 n+(5 n-1) x+2(3 n-2) x^{2}+6(n-1) x^{3}+4(n-1) x^{4}+(n-1) x^{5}
$$




$$
+F(x) \sum_{i=2}^{n-1} \sum_{j=1}^{i-1} x^{3(i-j)+1}
$$

where ,

$$
F(x)=1+4 x+6 x^{2}+4 x^{3}+x^{4},
$$

and

$$
\begin{aligned}
H\left(I_{n}\left(C_{6}\right) ; x\right) & =6 n+(7 n-1) x+(10 n-4) x^{2}+(11 n-8) x^{3}+10(n-1) x^{4}+8(n-1) x^{5} \\
& +4(n-1) x^{6}+(n-1) x^{7}+F(x) \sum_{i=2}^{n-1} \sum_{j=1}^{i-1} x^{4(i-j)+1},
\end{aligned}
$$

where

$$
F(x)=1+4 x+8 x^{2}+10 x^{3}+8 x^{4}+4 x^{5}+x^{6} \text {. }
$$

2. If $\mathrm{S}_{\mathrm{t}}, \mathrm{t} \geq 4$ is a star of order $\mathrm{t}$, then assuming that the vertex of introducing edge of each $S_{t}$ is an end vertex and by using Corollary 3.5,we get

$$
\begin{aligned}
H\left(I_{n}\left(S_{t}\right) ; x\right) & =n\left[t+(t-1) x+\frac{1}{2}(t-1)(t-2) x^{2}\right]+(n-1)\left[1+x+(t-2) x^{2}\right]^{2} x \\
& +\left[1+x+(t-2) x^{2}\right]^{2} \sum_{i=2}^{n-1} \sum_{j=1}^{i-1} x^{3(i-j)+1} \\
& =n t+(n t-1) x+\left[\frac{n}{2}(t-1)(t-2)+2(n-1)\right] x^{2}+(n-1)(2 t-3) x^{3} \\
& +2(n-1)(t-2) x^{4}+(n-1)(t-2)^{2} x^{5}+\left(1+x+(t-2) x^{2}\right)^{2} \sum_{i=2}^{n-1} \sum_{j=1}^{i-1} x^{3(i-j)+1} .
\end{aligned}
$$

3. If $\mathrm{W}_{\mathrm{t}}, \mathrm{t} \geq 4$, is a wheel of order $\mathrm{t}$ then, assuming that the vertex of introducing edge of each $\mathrm{W}_{\mathrm{t}}$ is a vertex of degree 3 and by using Corollary 3.5, we get

$$
\begin{aligned}
H\left(J_{n}\left(W_{t}\right) ; x\right) & =n\left[t+2(t-1) x+\frac{1}{2}(t-1)(t-4) x^{2}\right]+(n-1)\left[1+3 x+(t-4) x^{2}\right]^{2} x \\
& +\left[\left(1+3 x+(t-4) x^{2}\right]^{2} \sum_{i=2}^{n-1} \sum_{j=1}^{i-1} x^{3(i-j)+1}\right. \\
& =n t+(2 n t-n-1) x+\left[\frac{n}{2}(t-1)(t-4)+6(n-1)\right] x^{2}+(n-1)(2 t+1) x^{3} \\
& +6(n-1)(t-4) x^{4}+(n-1)(t-4)^{2} x^{5}+\left(1+3 x+(t-4) x^{2}\right)^{2} \sum_{i=2}^{n-1} \sum_{j=1}^{i-1} x^{3(i-j)+1} .
\end{aligned}
$$

\subsection{Wiener Index and Average Distance.}

The Wiener index of a connected graph $\mathrm{G}$ is denoted by $\mathrm{W}(\mathrm{G})$ and defined by $W(G)=\frac{1}{2} \sum_{u, v \in V(G)} d(u, v)$.

The name Wiener index for the quantity defined in (4.3.1) is usual in chemical literature, since Harold Wiener [8] in 1947 seems to be the first who considered it. Several mathematical authors obtained the Wiener index of many kinds of chain of cycle graphs $[2,10]$. The Wiener index of $\mathrm{G}$ can be obtained from the following formula $W(G)=\left.\frac{d}{d x} H(G ; x)\right|_{x=1}=\sum_{k \geq 1} k d(G, k)$,

where $\mathrm{H}(\mathrm{G} ; \mathrm{x})$ is the Hosoya polynomial of a connected graph $\mathrm{G}$. 
Consequently, the main distance or average distance of a connected graph $\mathrm{G}$, denoted by $\mu(G)$ and is defined by $\mu(G)=2 W(G) / p(p-1), p=|V(G)|$.

Finally, we define the Wiener index of any vertex $u$ in a connected graph $G$ by $W(u, G)=\left.\frac{d}{d x} H(u, G ; x)\right|_{x=1}=\sum_{k \geq 1} k d(u, G, k)$

From (4.3.2) and (4.3.4) and Corollary 2.5, we obtain the following corollary.

Corollary 4.3.1: For $n \geq 3$, we have.

$W\left(J_{n}(G)\right)=n W(G)+n(n-1)(H(u, G ; 1)-1) W(u, G)+\delta\left(\begin{array}{l}n \\ 3\end{array}\right)(H(u, G ; 1)-1)^{2}$.

Proof: $W\left(J_{n}(G)\right)=\left.\frac{d}{d x} H\left(J_{n}(G) ; x\right)\right|_{x=1}$

$=n W(G)+2(n-1)(H(u, G ; 1)-1) W(u, G)$

$$
\begin{aligned}
& +(H(u, G ; 1)-1)^{2} \sum_{i=2}^{n-1} \sum_{j=1}^{i-1} \delta(i-j)+2(H(u, G ; 1)-1) W(u, G) \sum_{i=2}^{n-1} \sum_{j=1}^{i-1} 1 \\
& =n W(G)+n(n-1)(H(u, G ; 1)-1) W(u, G)+\delta\left(\begin{array}{l}
n \\
3
\end{array}\right)(H(u, G ; 1)-1)^{2} .
\end{aligned}
$$

Now, from Corollary (4.3.1), we obtain the following corollary.

Corollary 4.3.2: For $n \geq 3$,we have:

1. $W\left(J_{n}\left(C_{2 m}\right)\right)=\frac{n}{3}\left(2 n^{2}+1\right) m^{3}-\frac{n}{3}\left(2 n^{2}-3 n+1\right) m^{2}+\frac{n}{6}\left(n^{2}-3 n+2\right) m, m \geq 2$.

2. $W\left(J_{n}\left(S_{t}\right)\right)=\frac{n}{3}\left(n^{2}+3 n-1\right) t^{2}-\frac{n}{3}\left(2 n^{2}+9 n-5\right) t+\frac{n}{3}\left(n^{2}+6 n-4\right), t \geq 4$.

3. $W\left(J_{n}\left(W_{t}\right)\right)=\frac{n}{3}\left(n^{2}+3 n-1\right) t^{2}-\frac{n}{3}\left(2 n^{2}+15 n-8\right) t+\frac{n}{3}\left(n^{2}+12 n-7\right), t \geq 4$.

But, from (4.3.2) and (4.3.4) and Corollary 3.5, we obtain the following corollary.

Corollary 4.3.3: For $n \geq 3$, we have:

$W\left(I_{n}(G)\right)=n W(G)+n(n-1) H(u, G ; 1) W(u, G)+(H(u, G ; 1))^{2}\left\{\delta\left(\begin{array}{l}n \\ 3\end{array}\right)+\left(\begin{array}{c}n+1 \\ 3\end{array}\right)\right\}$.

Proof: $\mathrm{W}\left(\mathrm{I}_{\mathrm{n}}(\mathrm{G})\right)=\left.\frac{\mathrm{d}}{\mathrm{dx}} \mathrm{H}\left(\mathrm{I}_{\mathrm{n}}(\mathrm{G}) ; \mathrm{x}\right)\right|_{\mathrm{x}=1}$

$$
\begin{aligned}
=n W(G)+(n-1) & \left\{(H(u, G ; 1))^{2}+2 H(u, G ; 1) W(u, G)\right\} \\
+ & (\mathrm{H}(\mathrm{u}, \mathrm{G} ; 1))^{2} \sum_{\mathrm{i}=2}^{\mathrm{n}-1} \sum_{\mathrm{j}=1}^{\mathrm{i}-1}\{(\delta+1)(\mathrm{i}-\mathrm{j})+1\}+2 \mathrm{H}(\mathrm{u}, \mathrm{G} ; 1) \mathrm{W}(\mathrm{u}, \mathrm{G}) \sum_{\mathrm{i}=2}^{\mathrm{n}-1} \sum_{\mathrm{j}=1}^{\mathrm{i}-1} 1 \\
= & n W(G)+n(n-1) H(u, G ; 1) W(u, G)+(H(u, G ; 1))^{2}\left\{\delta\left(\begin{array}{l}
n \\
3
\end{array}\right)+\left(\begin{array}{c}
n+1 \\
3
\end{array}\right)\right\} .
\end{aligned}
$$

Now, from Corollary (4.3.3), we obtain the following corollary.

Corollary 4.3.4: For $n \geq 3$, we have: 
1. $W\left(I_{n}\left(C_{2 m}\right)\right)=\frac{1}{3} n\left(2 n^{2}+1\right) m^{3}+\frac{2}{3} n\left(n^{2}-1\right) m^{2}, m \geq 2$.

2. $W\left(I_{n}\left(S_{t}\right)\right)=\frac{1}{2} n\left(n^{2}+2 n-1\right) t^{2}-n(3 n-1) t+n, t \geq 4$.

3. $W\left(I_{n}\left(W_{t}\right)\right)=\frac{1}{2} n\left(n^{2}+2 n-1\right) t^{2}-n(5 n-2) t+2 n, t \geq 4$. corollary.

Finally, from (4.3.3) and Corollaries 4.3.2 and 4.3.4, we have the following

Corollary 4.3.5: For $n \geq 3$, we have.
1. $\mu\left(J_{n}\left(C_{2 m}\right)\right)=\frac{m}{3}\left(n-1+\frac{2 m^{2}(2 n+1)-(n-1)}{n(2 m-1)^{2}+2 m-1}\right)$.
2. $\mu\left(J_{n}\left(S_{t}\right)\right)=\frac{2}{3}\left(n+3-\frac{t+4 n-1}{n t-n+1}\right)$.
3. $\mu\left(J_{n}\left(W_{t}\right)\right)=\frac{2}{3}\left(n+3-\frac{t+10 n-4}{n t-n+1}\right)$
4. $\mu\left(I_{n}\left(C_{2 m}\right)\right)=\frac{m}{3}\left(n+\frac{2\left(n^{2}-1\right)+n+m}{2 m n-1}\right)$.
5. $\mu\left(I_{n}\left(S_{t}\right)\right)=n+2-\frac{t^{2}+(5 n-4) t-2}{n t^{2}-t}$.
6. $\mu\left(I_{n}\left(W_{t}\right)\right)=n+2-\frac{t^{2}+(9 n-6) t-4}{n t^{2}-t}$.

\section{Conclusions}

In this paper, we generalized the operation of constructing vertex identified and edge introducing graphs obtained from two graphs to those obtained from a sequence of n pairwise vertex disjoint connected graphs $G_{1}, G_{2}, \ldots ., G_{n}$. Using Gutman's Theorems n times in each case, we obtained Hosoya polynomials of such generalized graphs.

Moreover, we obtained Hosoya polynomials, Wiener indices and average distance for generalized vertex identified and edge introducing graphs when every $G_{i}, 1 \leq i \leq n$, is isomorphic to a special graph which has important applications in Chemistry. 


\section{$\underline{\text { REFERENCES }}$}

[1] Ashrafi , A. R. and Yousefi , S. ; (2007), " Computing the Wiener index of a TUC4C8(S) nanotorus", MATCH , Vol. 57 , No. 2, pp. 403-410 .

[2] Balarishnan, R. and Ranganathan, K. ; (2000), "A Textbook of Graph Theory", Springer-Verlage New York, Inc.

[3] Buckly, F. and Harary , F. ;(1990), "Distance in Graphs", Addison-Wesley, New York.

[4] Dobrynin, A.A.; (1999), "A simple formula for calculation of the Wiener index of hexagonal chains", Computers and Chemistry Program on, Vol.23, pp.43-48.

[5] Douglas , B. W . ; (2002), "Introduction to Graph Theory", $2^{\text {nd }}$ edition, Indian Branch, 482 F.I.E. , India.

[6] Gutman, I. ; (1993), "Some properties of the Wiener polynomial", Graph Theory Notes of New York; XXV. The New York Academy of Sciences 13-17.

[7] Hosoya , H. ; (1988), "On some counting polynomials in Chemistry", Discrete Applied math.,19,239-257.

[8] Li, X. , Wang, G. , Bian, H. and Hu, R.; (2012), " The Hosoya polynomial decomposition for polyphenyl chains", MATCH Vol. 67 , No. 2, pp. 357-368.

[9] Rao, N.P. and Prasanna , A.L. ; (2008)," On the Wiener index of pentachains " , Applied Math. Scs., Vol. 2 , No. 49, 2443-2457.

[10] Saeed, W.A.M. ; (1999). "Wiener Polynomials of Graphs", Ph.D. Thesis , Mosul University.

[11] Sagan, B.E., Yeh , Y.N. and Zhang, P.; (1996), "The Wiener polynomial of a graph, International J.Quantum Chem.,60, No.5, 959-969. 